\title{
Ad hoc posterior tibial vessels perforator propeller flaps for the reconstruction of lower third leg soft- tissue defects
}

\author{
Thalaivirithan Margabandu Balakrishnan, Jayagosh Ramkumar, Janardhanan Jaganmohan \\ Department of Plastic and Faciomaxillary Surgery, Madras Medical College, Chennai, Tamil Nadu, India
}

Address for correspondence: Dr. Thalaivirithan Margabandu Balakrishnan, Old No 15, New No 10, Thiruvalluvar Street, Kodambakkam, Chennai - 600 024, Tamil Nadu, India. E-mail: thalaiviri.b@gmail.com

\section{ABSTRACT}

Introduction: Lower third leg soft tissue defects with anatomical and pathological constraints are posing formidable challenges to reconstructive surgeon. Aim: This retrospective study was conducted to assess the effectiveness of ad hoc posterior tibial vessels perforator-propeller flaps for the reconstruction of small and medium sized soft tissue defects in the lower third leg. Patients and Methods: 22 patients (16 were males and 6 were females) were involved in this study between period of January 2012 and December 2016. We followed the protocol of initial non delineating exploratory incision made to find out single best perforator in all patients. All the defects in leg reconstructed with adhoc posterior tibial vessel propeller flaps. Results: All 22 flaps survived well. All in an average of 13 months follow up period, had pain free walking, with minimal scarring and acceptable aesthesis at the reconstruction sites with no need for any secondary procedure. Conclusion: With inability of preoperatively dopplering the perforators in the lower third leg region, the exploratory posterior nondelineating incision was used in all cases to secure the single best perforator for the propeller flaps. Thus adhoc posterior tibial vessel propeller flaps are dependable, easily adoptable for the reconstruction of soft tissue defects of the lower third leg region.

\section{KEY WORDS}

Ad hoc posterior tibial vessel propeller flaps; exploratory non-delineating incision; extensile steps of skeletoniszation of perforator

\section{INTRODUCTION}

ower third leg soft- tissue defects are challenging the skills of reconstructive surgeon. The anatomical constraints for the reconstruction are the following

\begin{tabular}{|l|l|}
\hline \multicolumn{2}{|c|}{ Access this article online } \\
\hline Quick Response Code: & Website: \\
\hline & www.ijps.org \\
\hline & Dol: \\
\hline
\end{tabular}

(1) paucity of loose tissues in the vicinity (so local flaps cannot be raised easily without donor site morbidity); (2) subcutaneous bone and its prominences are abundant in this region (therefore not only with low energy injuries

This is an open access article distributed under the terms of the Creative Commons Attribution-NonCommercial-ShareAlike 3.0 License, which allows others to remix, tweak, and build upon the work non-commercially, as long as the author is credited and the new creations are licensed under the identical terms.

For reprints contact: reprints@medknow.com

How to cite this article: Balakrishnan TM, Ramkumar J, Jaganmohan J. Ad hoc posterior tibial vessels perforator propeller flaps for the reconstruction of lower third leg soft- tissue defects. Indian J Plast Surg 2017;50:281-7. 
they get exposed, but also devitaliszation of fracture fragment occurs quite easily because of wider periosteal stripping with loss of overlying soft tissue); (3) muscles in this region are tendonous (therefore muscle flaps are not available, tendons will get exposed easily with peninsular pedicled flaps and also potentially can predispose to unstable scar with skin grafting of secondary defect); (4) normally also relatively decreased regional blood supply here (therefore, peninsular pedicled flap business ends are more likely to have sublethal ischemia); (5) the cutaneous perforators are short and straight, overlies the source vessels in this region (therefore, difficulty in marking them pre-operatively by using hand- held Doppler) [Figure 1]; (6) the small circumference of the lower third leg segment (so any pedicled peninsular flaps may easily impinge on the lymphatic drainage, and potentially could precipitate the circumferential wound); and (7) finally distal ankle and foot regions are pressure bearing promontory (so they are all poor donor sites of "'like tissue'). Pathological constraints are degloving injuries in this region, which mars the prospectus of all perforator/propeller flaps and distally based pedicled flaps. The post- traumatic vascular disease (PTVD) $)^{[1]}$ of chronic wound renders the local and regional vessels poor recipients for the microvascular reconstruction which are considered as the first choice for reconstruction of lower third leg soft-tissue defects. All these anatomical and pathological constraints prevailing in the lower third leg soft-tissue defects narrows the reconstructive armamentarium of the surgeon to very few choices. Conventional or flow- through free flap for the delayed primary reconstruction of acute wounds and free flaps with cross-leg construct or microvascular loops ${ }^{[1]}$ for chronic wounds are worn- down choices. But later, envisage microvascular anastomotic expertise. On the other hand, in the lower third leg small- and medium-sized soft-tissue defects without any degloving injury, the

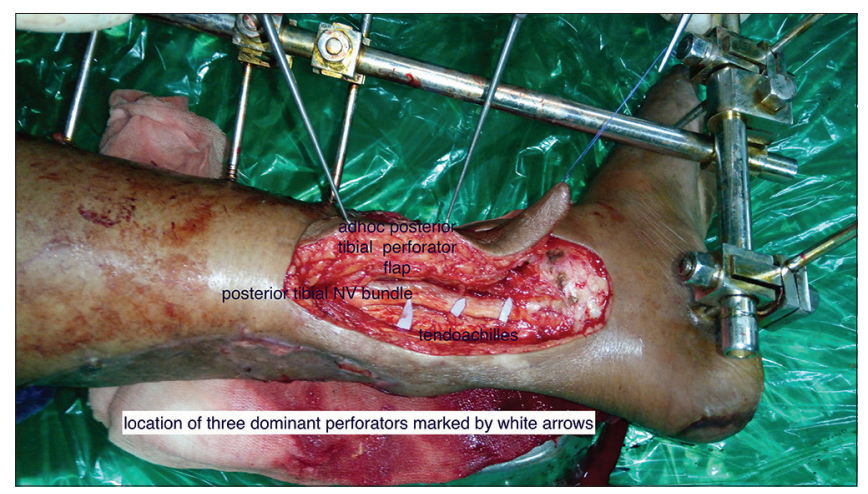

Figure 1: Short and straight cutaneous perforators from the posterior tibial vessels and they directly overlies the neurovascular bundle perforator propeller flaps are the authors preferred choice. These are basically islanded fascio cutaneous flaps have unique versatility that it has two unequal blades which can rotate about the dissected cutaneous single best perforator - the pivot point through $180^{\circ}$ in such a way that large blade fills the primary defect. The secondary defect is partially covered by the small blade of the propeller flap. They are preferred because they are microvascular surgery without microvascular anastomosis. They have reduced or nil donor site morbidity. Since the large blade of the flap overlies the proximal calf region, the resultant secondary defects can be closed primarily or uneventfully skin grafted. They have robust blood supply. They do not produce standing cones that occur invariably with any other peninsular pedicled flaps. They spare all the cutaneous nerves, large named subcutaneous veins and source axial vessels. Therefore, in this region, the perforator propeller flaps can be based on the perforators arising from posterior tibial, anterior tibial and peroneal vessels. However, the propeller flaps based on the posterior tibial vessels are preferred choice because of the following four reasons

1. Posterior tibial vessel and its perforators are safe guarded well in any trauma to lower third leg which causes the soft- tissue defects with exposed fracture fragments, as it lies away from the tibial bone, whereas the anterior tibial very close to the tibial malleolus and peroneal runs close to fibula

2. A minimum of $1.5 \mathrm{~cm}$ to $2 \mathrm{~cm}$ requirement length for gracious spiral turn without acute twist or kinking of pedicle during $180^{\circ}$ rotation is possible with posterior tibial vessels lying deeply beneath the fascia between the flexor digitorum longus and tendoachilles up to the proximal end of laciniate ligament. The subfascial peri perforator dissection facilitate to attain this required pedicle length

3. Because most of the time they need to cover the anteromedial exposed surface of tibia the propeller flaps based on the posterior tibial vessels easily does the job without having to pass over the shin promontory which other source vessel based propeller flaps have to do

4. In the middle third and proximal third areas, the perforators arising from posterior tibial vessels can be pre-operatively located by hand- held Doppler because the source vessels lies deeply. Whereas, in the lower third leg segment the perforators from posterior tibial have short straight course approximately $2 \mathrm{~cm}$ to reach the fascia and also overlies the source vessels [Figure 1]. Therefore, here the pre-operative Doppler mapping will 
not help in correct location of perforator. So initially, these perforators have to be explored by posterior non-delineating incision and then choose the single best perforator, which is going to be the pivot point of these propeller flaps. Then, the final dimensions of the flaps are designed according to the bio geometry of the propeller flaps based on the single- best perforator. Therefore, these flaps are aptly (cut as you go) called ad hoc posterior tibial propeller flaps. ${ }^{[2]}$

This article reports the aesthetic and final outcome of ad hoc posterior tibial vessel propeller flaps for the reconstruction of lower third leg small- and medium- sized soft- tissue defects in 22 patients.

\section{Aim}

The aim of this study is to analysze the outcome of reconstruction of small- and medium- sized lower third leg soft- tissue defects using the ad hoc posterior tibial vessel perforator propeller flaps.

\section{PATIENTS AND METHODS}

Twenty-two patients (16 were male and 6 were female) were involved in this study between January 2012 and December 2016. We followed the protocol of initial non- delineating exploratory incision made to find out single best perforator (based on the presence of venae comitantes, size of the fascial defect, size of the vessel after spraying lignocaine measured at fascial level) in all patients. All the defects in leg reconstructed with ad hoc posterior tibial vessel propeller flaps with bio geometry described by Teo. ${ }^{[3]}$

\section{Selection criteria}

1. All those with lower third leg soft- tissue defects with good general condition, and unaffected peripheral circulation were chosen

2. The maximum width of the soft- tissue defect should be less than or equal to the one third of circumference of the lower third leg (mainly small- and medium- sized defects were chosen)

3. Those without any degloving injury element were chosen.

\section{Exclusion criteria}

Following patients were excluded from the study

1. All those with peripheral vascular disease

2. All those with comorbid medical illnesses such as diabetes mellitus, collagen vascular diseases, and so on
Table 1: Demographic data and intraoperative data

\begin{tabular}{|c|c|c|c|c|}
\hline Number & Age/sex & Cause & $\begin{array}{c}\text { Single best } \\
\text { perforator size }(\mathrm{mm})\end{array}$ & Size of flap* \\
\hline 1 & 22/female & Trauma & 1 & $10 \times 4$ \\
\hline 2 & 35/female & Trauma & 2 & $8 \times 4$ \\
\hline 3 & 43/male & Trauma & 1.5 & $12 \times 3$ \\
\hline 4 & 24/male & Trauma & 1.7 & $8 \times 4$ \\
\hline 5 & 36/female & Trauma & 1.2 & $9 \times 3.5$ \\
\hline 6 & $18 /$ male & Trauma & 1.3 & $10 \times 3$ \\
\hline 7 & $50 /$ male & $\begin{array}{l}\text { Exposed } \\
\text { implant }\end{array}$ & 1.5 & $12 \times 3.5$ \\
\hline 8 & $57 /$ male & Trauma & 1 & $9 \times 3.5$ \\
\hline 9 & 20/male & $\begin{array}{l}\text { Exposed } \\
\text { implant }\end{array}$ & 1.5 & $7.5 \times 3$ \\
\hline 10 & $14 /$ male & Trauma & 1.8 & $12 \times 5$ \\
\hline 11 & 13/female & Trauma & 1 & $13 \times 4$ \\
\hline 12 & 23/female & Trauma & 1.4 & $8 \times 4$ \\
\hline 13 & $36 /$ male & Trauma & 2.1 & $9 \times 5$ \\
\hline 14 & $34 /$ male & Trauma & 1.4 & $10 \times 5$ \\
\hline 15 & $42 /$ male & Trauma & 1.7 & $8 \times 4$ \\
\hline 16 & 63/female & Trauma & 1.8 & $9 \times 4$ \\
\hline 17 & 20/male & Trauma & 1.5 & $8 \times 4$ \\
\hline 18 & $59 /$ male & Trauma & 1.3 & $9 \times 3$ \\
\hline 19 & $27 /$ male & Trauma & 1.4 & $9 \times 4$ \\
\hline 20 & $20 /$ male & Trauma & 1.5 & $12 \times 3$ \\
\hline 21 & 19/male & Trauma & 1.7 & $10 \times 3$ \\
\hline 22 & 50/female & $\begin{array}{l}\text { Snake } \\
\text { bite }\end{array}$ & 1 & $11 \times 3.5$ \\
\hline
\end{tabular}

*Size of the defect was $1.75 \mathrm{~cm}$ smaller in greatest dimension of flap and $0.5 \mathrm{~cm}$ smaller in the small dimension of flap in all cases

3. All those with composite defect of the lower third leg

4. All smokers who fail to quit the habit 4 weeks before the procedure

5. All those lower third leg soft- tissue defects with width greater than the one third of circumference and those with defects closed by peroneal and anterior tibial vessel perforators.

Demographic data of the patient are given in Table 1.

\section{Pre-operative preparation}

A written informed consent was taken from all these patients. X- rays were taken in all cases to rule out any osteomyelitis. No pre-operative Doppler examination was done in any cases. None of the cases had any other radiological imaging of vessels or perforator.

\section{Surgical technique}

Non-exsanguinating pneumatic tourniquet was used in all cases, which helped in the easy identification of the perforators. The first step was the thorough excisional debridement using two drapes and instruments system followed by thorough lavage of the wound. Dissection started with posterior non-delineating incision and all 
the septofasciocutaneous perforators from posterior tibial vessels were identified in subfascial dissection. The peri perforator dissection was carried out from the source vessel up to the deep fascial strip by dividing and ligating the small tendonous branches (not to diathermise them as they produce the thrombosis in the perforator vessels), and all fascial strands were cleaned around the cytoskeleton that contained the perforator artery and venae comitantes and possibly the lymphatics. This should result in the minimum of $1.5 \mathrm{~cm}$ to $-2 \mathrm{~cm}$ requirement length, which could permit the gracious spiral turn without acute twist or kinking of pedicle during $180^{\circ}$ rotation of flap. The single best perforator was not chosen in all cases by trial soft clamping the other perforators and watching the perfusion of the flap as mentioned by many studies. ${ }^{[4]}$ This is because the only after choosing the single best perforator the siting, designing and raising of flap by the whole delineating incision could be completed. Although in several studies applying the soft intestinal clamp across the non-delineated potential boundaries of the flap was mentioned, it envisage unnecessary extra dissections and it was cumbersome procedure..$^{[1,2,5]}$ However, the author in choosing the single best perforator followed the following criteria

1. A large size perforator with prominent venae comitantes

2. Passing through wide fascial defect

3. Pulsatile prominently after release of tourniquet and spraying with the $2 \%$ xylocaine

4. One that is strategically located close to the defect, but at the same time, it should not be involved by the PTVD, scar and not encased in granulation tissue.

Once the single best perforator was secured the flap was designed in such a way that it would be propelled tension free to the distal most edge of the primary defect.

In the bio geometry of propeller flap author assiduously followed several steps

1. At the waist of the propeller flap close to the pivot point or the location of single best perforator, there must be equal flap dimension on either side because this avoids the tension on the pedicle during inset. Eccentric location of the perforator causes tension on the pedicle during the inset ${ }^{[3]}$

2. Rest of the flap bio geometry was along the lines described by Teo. ${ }^{[4]}$ The location of the single best perforator was the pivot point. From the pivot point, the farthest edge of the primary defect was measured and to this dimension $1.5 \mathrm{~cm}$ was added (this is the allowance for the primary contraction of flap) to get the length of the large blade of the propeller flap. The distance between the pivot point and the nearest edge of the defect was the length of the short blade of the propeller flap. The width of the flap was the width of the primary defect and plus $0.5 \mathrm{~cm}$ (as an allowance for the primary contraction of the flap)

3. After release of tourniquet and completely islanding the flap, it was left in orthotopic position to get matured and stabilised for 10 min before any primary movement of flap. During this time, haemostasis was secured and also perfusion of flap was observed with application of topical vasodilators to the pedicle

4. To determine whether clock wise or anticlockwise rotation as primary movement of flap two factors were taken in to consideration (a) which rotation was shortest? (It was determined by the siting of flap in relation to primary defect) and (b) which rotation brings the venous congestion? Sometimes in extremes of $180^{\circ}$ rotation irrespective of the direction of rotation, there will be venous congestion and this would be always due to some unrecogniszed residual fascial strands in the cytoskeleton of the pedicle, which became obvious on rotation and causing compression on the low-pressure venae comitantes of the pedicle. Once these residual fascial strands were released the venous congestion disappears. These are extensile steps in the skeletoniszation of the perforator from source vessel to fascia

5. Tension- free inset was ensured every time. Even mild tensions cause the collapse of sub dermal venous plexus and cause the venous congestion along the suture line. This is specifically common in the propeller flaps where the venous circulation is centripetally directed towards the single best perforator.

Suction drains were used in all cases.

All surgeries were performed under $\times 4$ loupe magnification.

\section{Post-operative}

All were given cottony fluffy dressing with window to monitor the flap. On an average of 13- months follow- up, patients were assessed for the scar status, flap cover stability, distal lymphedema, functional status such as like pain- free walking. 


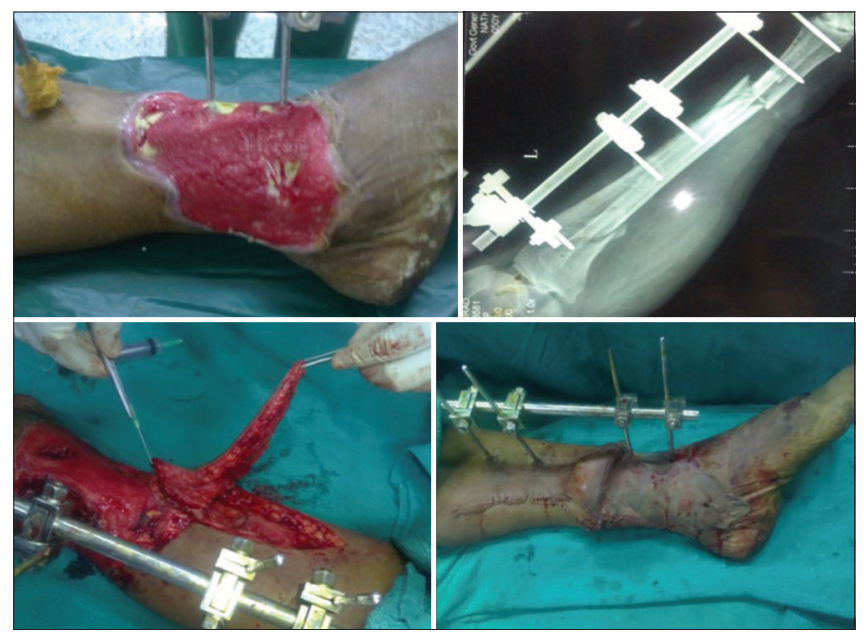

Figure 2: The Case 1 ad hoc posterior tibial vessel propeller flap for medium-sized defect in the lower third leg region (pre- and post-operative pictures)

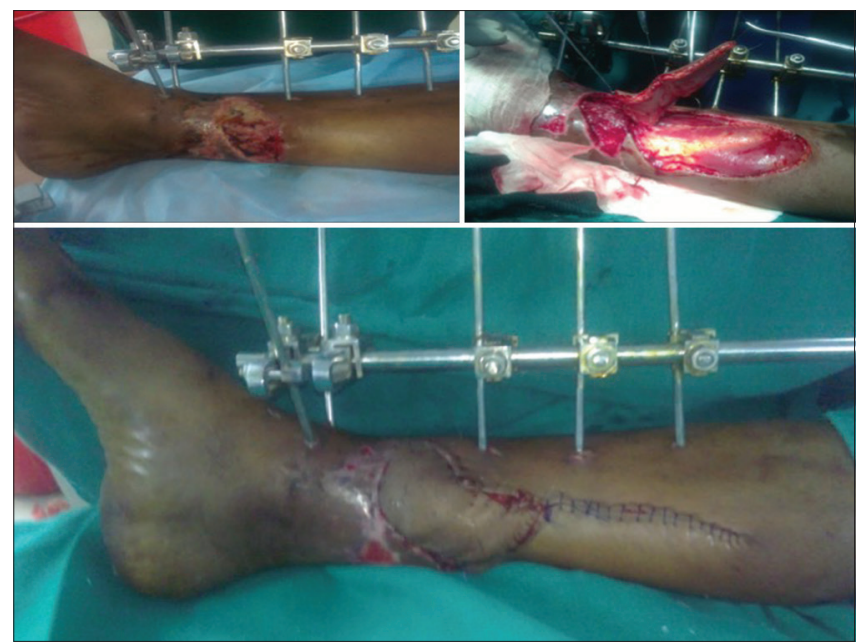

Figure 4: The Case 3 with ad hoc posterior tibial vessel perforator propeller flap with secondary defect closed primarily (pre- and post-operative pictures)

\section{RESULTS}

The average size of the flap was $35.09 \mathrm{~cm}^{2}$ [Figures 2-6]. The average size of single best perforator was $1.3 \mathrm{~mm}$. Age group of these patients were ranging from 13 to 63 years. None of the flap failed and one patient developed post-operative hematoma and drained. One patient had partial superficial epidermolysis and dermal healing followed uneventfully. No other complication encountered. There was aesthetically acceptable scar at donor site in all cases. No other secondary procedures were needed (like flap thinning or correcting standing cone). All patients assumed pain-free walking in an average 13-month follow-up period. Great saphenous vein and saphenous nerve were preserved in all cases when they were intact. In 15 cases, skin grafting was done for the secondary raw areas. Rest had primary

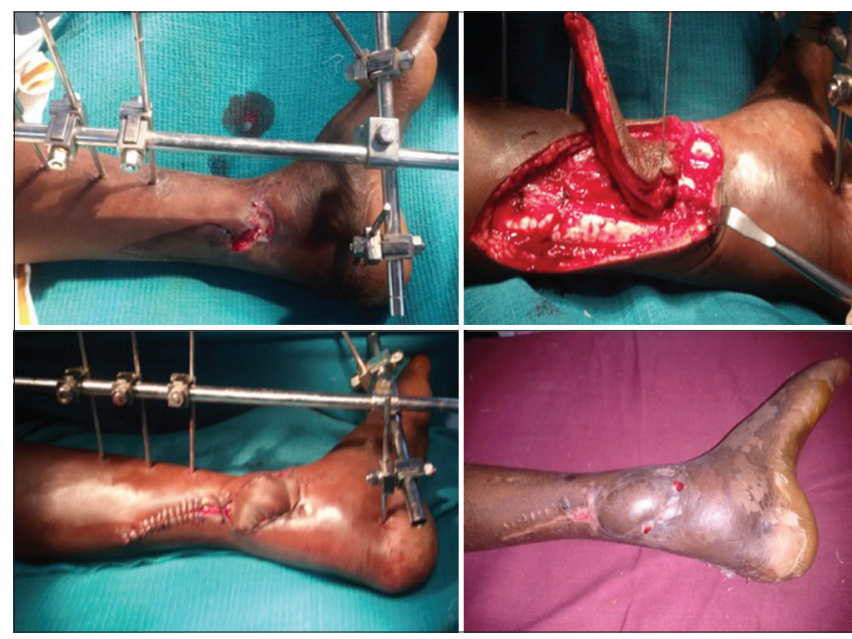

Figure 3: The Case 2 with ad hoc posterior tibial vessel perforator propeller flap for the reconstruction lower third leg defects (pre- and post-operative pictures)

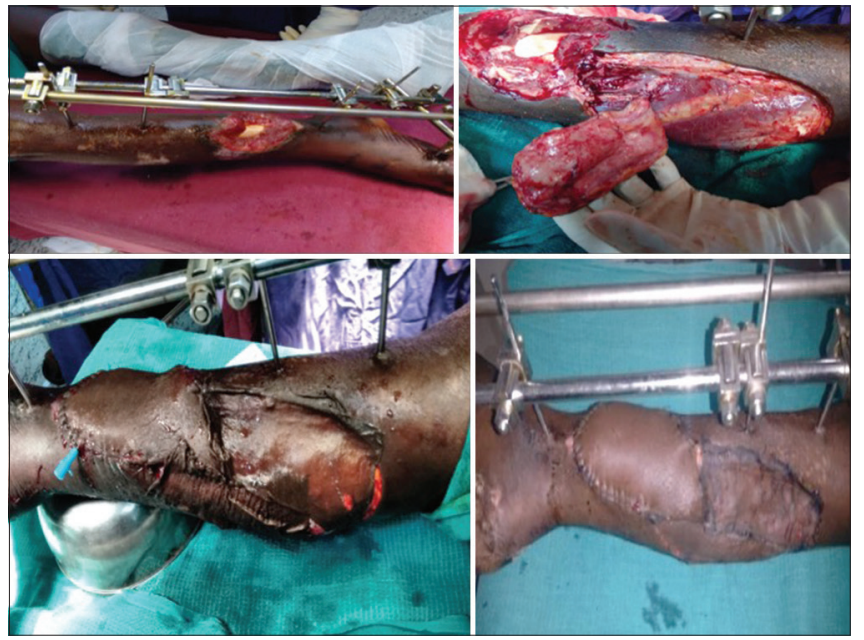

Figure 5: The Case 4 with ad hoc perforator propeller flap for the medium-sized defect in the lower third leg region (pre- and post-operative pictures)

closure. All the cases were completed in an average of $1 \mathrm{~h}$ and $30 \mathrm{~min}$.

\section{DISCUSSION}

The propeller flaps were originally conceived for the lower third leg soft-tissue defects. ${ }^{[3]}$ Teo perfected the technique of propeller flaps..$^{[3-5]}$ Hyakusoku et al. ${ }^{[6]}$ were the first proponents of propeller flaps but they all, had subcutaneous pedicle. The perforosomes of posterior tibial vessels are located with an axial line drawn craniocaudally on the medial aspect of leg 2- $\mathrm{cm}$ posterior and parallel to the anteromedial borer of tibia. All these perforators pass through the septum (septofasciocutaneous) between the flexor digitorum longus and soleus muscles (in the middle 


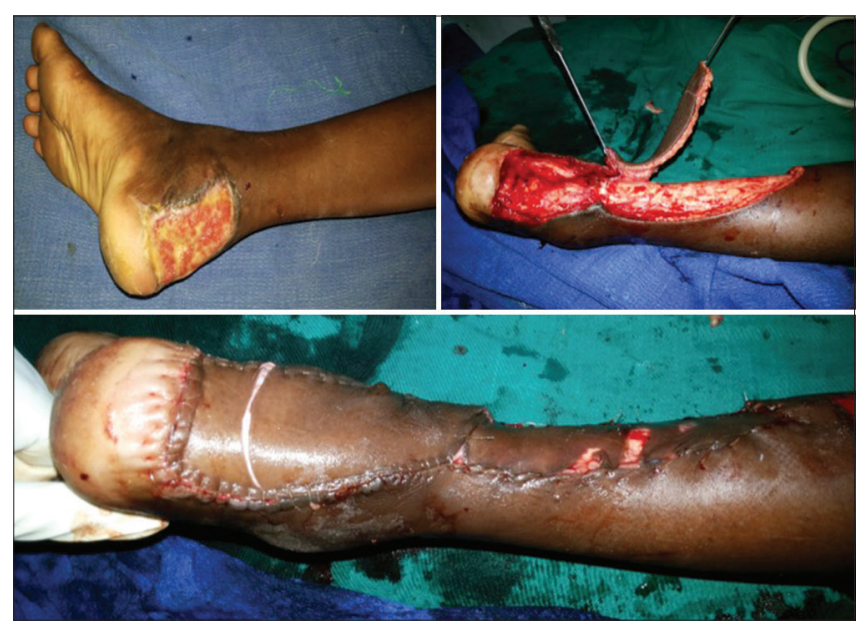

Figure 6: The Case 5 with tendoachilles defect covered by ad hoc posterior tibial vessel perforator propeller flap (pre- and post-operative pictures)

third leg) to reach the skin except in the proximal $10 \mathrm{~cm}$, they pass through soleus (musculocutaneous). In the lower third leg, they are having short and straight course through the septum between the tendoachilles and tendon of flexor Digitorum longus. All these perforosomes were interconnected by the anastomotic direct and indirect linking vessels in the suprafascial plane. ${ }^{[7]}$ Among the 21 angiosomes ${ }^{[7]}$ described in the lower limb, uniquely at the lower third leg region, there exist short and straight perforators directly overlying the source posterior tibial vessels. Therefore, the routine pre-operative marking of the perforators is not practical here because the Doppler acoustic signals of perforators cannot be distinguished from the source vessels. Hence, the perforator flaps based on the posterior tibial vessels in this region are named as ad hoc perforator propeller flaps, wherein the perforators are first exposed by exploratory posterior non-delineation incision and then single best perforator was selected. Hence, the author's technique provided the definitive way of harvesting robust vascularity, reliable propeller flaps. Pre-operative colour Doppler examination is not reliable and not cost-effective again. Pre-operative multiple detector computed tomography (CT) scan or the three-dimensional CT angiography with methodical interactive medical imaging with control system which can delineate easily the location, size, course, branching and anastomotic pattern of the perforators and also delineates the anatomy of source vessels. However, these expensive gadgets were not available uniformly in all places. Therefore, this technically less demanding procedure is a boon for the reconstructive surgeon. In addition, these propeller flaps are local flaps with good color, texture, and thickness match; are excellent form of reconstruction with predictable outcome and that too in the single stage. Bekara et al..$^{[8]}$ meta-analysis study involving 40 articles on the perforator flaps though analysed various factors, there was no specific mention about any technicalities in the posterior tibial vessel based propeller flaps. Author in this article describes the extensile steps in the skeletonisation and preparation of perforator from posterior tibial vessels of the lower third leg region. The ergonomics followed by the authors in the selection, siting, designing and execution of flap can exonerates one from the complications and failure. In another relatable study by Don et al. ${ }^{[9]}$ where there was only five posterior tibial vessel based propeller flaps were done and there was no mention about these ad hoc propeller flaps. Hence, the author's study on the ad hoc posterior tibial perforator propeller flap is first of its kind throwing light on more technical aspects for safe harvest of these flaps.

One another large relatable study by Quaba et al..$^{[10-12]}$ was conducted 66 patients in 5-year duration. Their complication rate was (complete loss of flap necessitating another reconstructive procedure) $7.5 \%$. They contributed three reasons for the flap loss - (1) diabetes mellitus, (2) peripheral vascular disease and (3) unrecognised degloving injury. Although the author's study was small sized, the reason for the absent of major complications could be attributed to (1) careful patient selection (see the selection and exclusion criteria), (2) choosing the single best perforator with good venae comitantes and subsequently locating the flap away from the zone of injury (3) diligently following the extensile meticulous steps in the periperforator dissection (mentioned in the surgical technique) to get rid of last lurking fascial strand that causes the venous congestion. All the flaps were rotated through $180^{\circ}$. Never leave the operating room leaving the congested perforator propeller flaps hoping to improve on its own, which never happens. If the venous congestion appears, it always happens on the table. Hence, it has to be addressed effectively at the time of primary surgery. All these contributed to the good results in our study.

The benefits of ad hoc posterior tibial perforator/propeller flaps are (1) these are microvascular operations minus the microvascular anastomosis, (2) robust and dependable blood supply, ${ }^{[13]}$ (3) With preservation of source vessels and sparing the saphenous nerve and vein the donor site morbidity is minimal, (4) relatively constant location of perforators with constant anatomy and (5) pre-operative Dopplering is not needed. 


\section{CONCLUSION}

Ad hoc posterior tibial perforator flaps extricate the reconstructive surgeon from the peninsular pedicled flaps strict biogeometry shackles, by providing easy 'cut as you go' after securing the single best perforator. These are new addition in the armamentarium of reconstructive surgeon and are dependable, less technically demanding, easily adoptable flaps.

\section{Declaration of patient consent}

The authors certify that they have obtained all appropriate patient consent forms. In the form the patient(s) has/have given his/her/their consent for his/her/their images and other clinical information to be reported in the journal. The patients understand that their names and initials will not be published and due efforts will be made to conceal their identity, but anonymity cannot be guaranteed.

\section{Financial support and sponsorship} Nil.

\section{Conflicts of interest}

There are no conflicts of interest.

\section{REFERENCES}

1. Mambally SR, Santha KK. Utility of arteriovenous loops before free tissue transfer for post-traumatic leg defects. Indian J Plast Surg 2015;48:38-42.
2. Waterston SW, Quaba O, Quaba AA. The ad hoc perforator flap for contracture release. J Plast Reconstr Aesthet Surg 2008;61:55-60.

3. Teo TC. Reconstruccion de la extremidad inferior con colgaios de perforantes locales [Perforator local flaps in lower limb reconstruction]. Cir Plas Iberolatinoam 2006;32:287-92.

4. Teo TC. The propeller flap concept. Clin Plast Surg 2010;37:615-26, vi.

5. Quaba O, Quaba A. Pedicled perforator flaps in the lower limb. Semin Plast Surg 2006;20:103-11.

6. Hyakusoku H, Yamamoto T, Fumiiri M. The propeller flap method. Br J Plast Surg 1991;44:53-4.

7. Geddes CR, Tang M, Yang D, Blondeel P, Morris SF, Hallock $\mathrm{GG}$, et al. Anatomy of the integument of the lower extremity. In Geddes CR, Tang M, Yang D, Blondeel P, Morris SF, Hallock GG, et al, editors. Perforator flaps: Anatomy, technique and clinical applications. St Louis (MO): QMP; 2006. p. 541-78.

8. Bekara F, Herlin C, Mojallal A, Sinna R, Ayestaray B, Letois F, et al. A systematic review and meta-analysis of perforator-pedicled propeller flaps in lower extremity defects: Identification of risk factors for complications. Plast Reconstr Surg 2016;137:314-31.

9. Dong $\mathrm{KX}, \mathrm{Xu} Y \mathrm{Y}$, Fan $\mathrm{XY}, \mathrm{Xu} \mathrm{LJ}$, Su XX, Long $\mathrm{H}$, et al. Perforator pedicled propeller flaps for soft tissue coverage of lower leg and foot defects. Orthop Surg 2014;6:42-6.

10. Quaba AA. Local flaps. In: Court-Brown CM, McQueen MM, Quaba AA, editors. Management of Open Fractures. London: Martin Dunitz Publishers; 1996. p. 195-209.

11. Court-Brown CM, Quaba AA. The relationship between plastic surgery and orthopaedic trauma surgery. In: Court-Brown CM McQueen MM, Quaba AA, editors. Management of Open Fractures. London: Martin Dunitz Publishers; 1996. p. 157-64.

12. Erdmann MW, Court-Brown CM, Quaba AA. A five year review of islanded distally based fasciocutaneous flaps on the lower limb. Br J Plast Surg 1997;50:421-7.

13. Rubino C, Ramakrishnan V, Figus A, Bulla A, Coscia V, Cavazzuti MA, et al. Flap size/flow rate relationship in perforator flaps and its importance in DIEAP flap drainage. J Plast Reconstr Aesthet Surg 2009;62:1666-70. 